\title{
Urgent endoscopy in lower gastrointestinal bleeding
}

Jensen DM, Machicado GA, Jutabha R, et al. Urgent colonoscopy for the diagnosis and treatment of severe diverticular hemorrhage. N Engl f Med 2000;342:7882 .

\section{Background}

Little has been published regarding the role of endoscopic therapy in acute lower gastrointestinal bleeding (haematochezia) in contrast with the almost universal adoption of endoscopic methods of achieving haemostasis for upper gastrointestinal bleeding. The authors examined the role of such intervention for acute colonic diverticular haemorrhage.

\section{Design}

Two prospective studies of patients admitted to two centres with severe haematochezia and diverticulosis, all of whom underwent urgent colonoscopy (within 6-12 hours of admission) after cleansing the bowel. The first study was of 73 patients from 1986-1992 and those who rebled after diagnostic colonoscopy underwent hemicolectomy. The second study included 48 cases, studied from 1994 to 1998, who underwent endoscopic haemostatic interventions if active bleeding from diverticular disease was found. Bowel cleansing was by the oral or nasogastric route, using 5-6 litres of purge over 3-4 hours, followed within an hour by colonoscopy at the bedside under conscious sedation. Cases without evidence of diverticular disease were excluded from the study.

\section{Results}

Definite signs of acute diverticular haemorrhage were found in $17 / 73(23 \%)$ of the first study group (diagnostic colonoscopy only), nine rebled, and six underwent hemicolectomy. In contrast with 10/48 of the latter group with definite signs of diverticular haemorrhage, all underwent endoscopic treatment (injection of epinephrine or bipolar coagulation) with no recurrence of bleeding or need for surgery. A definite cause of bleeding was identified in all of the first cohort, most common diagnoses other than diverticular haemorrhage being angioma, cancer, polyps, or ischaemic colitis. In the later cohort, no bleeding lesion was identified in 14/48 cases, other causes of bleeding being similar to the previous study. Patient characteristics, endoscopic stigmata of haemorrhage, and follow up were similar for both groups. Recurrent bleeding after first colonoscopy and median time to discharge was significantly less in the endoscopically treated group. However, numbers were small and great significance cannot be attached to these differences.

\section{Conclusions}

Urgent endoscopic and treatment of acute diverticular haemorrhage appears to be a practical and simple method of controlling a significant proportion of patients with lower gastrointestinal haemorrhage. It may well avoid the need for surgery in a predominantly elderly patient group. Further larger randomised trials are required to evaluate this treatment option.

\section{Comment}

Endoscopy plays an important role in the management of acute gastrointestinal bleeding. Therapeutic endoscopy in upper gastrointestinal bleeding helps to achieve haemostasis, decreases the risk of rebleeding and the need for surgery. Jensen et al have examined whether urgent diagnostic and therapeutic endoscopy in lower gastrointestinal bleeding results in similar benefits. ${ }^{1}$ They report that the endoscopic stigmata of haemorrhage seen in upper gastrointestinal bleeding (that is, active bleeding, non-bleeding visible vessels, or adherent clots) also appear to predict the risk of rebleed in patients who have bled from diverticular disease. Therapeutic intervention with epinephrine injection or bipolar coagulation in patients with these stigmata was beneficial with decreased rebleeding rates, leading to avoidance of surgical intervention.

The study only examined patients with identifiable diverticular disease and haematochezia. Although diverticular bleeding is the commonest cause of acute lower gastrointestinal bleeding, the majority of these $(70-80 \%)$ stop bleeding spontaneously ${ }^{2}$ and the mortality of diverticular bleeding is low ${ }^{3}$ compared with bleeding due to peptic ulceration. The benefits in this study need to be interpreted in the context of this subgroup of patients with a relatively "benign" clinical course and outcome compared with upper gastrointestinal bleeding.

The study used a sulphate purge until the clearance of stool, blood, and clots was achieved before performing colonoscopy within 6-12 hours of admission. It was effective and appeared to be well tolerated in all patients in the study. Despite this, we have some concern about the tolerability, efficacy, or indeed safety of urgent bowel preparation in acute lower gastrointestinal bleeding. It is conceivable that a large volume purge may exacerbate lower gastrointestinal bleeding in some patients. ${ }^{4}$ The potential problems with voluminous bowel preparation include clinically significant fluid retention, which occurred in $4 \%$ of patients in their previous study. ${ }^{5}$

In the study, $17(23 \%)$ patients with diverticulosis and haematochezia in the first group and $10(21 \%)$ patients in the second group had signs of diverticular haemorrhage. In routine practice, the incidence of identifiable bleeding lesions during endoscopy is likely to be lower and the incidence of treatable lesions is likely to be even lower. The therapeutic techniques used in this study need to be compared with other options for safety and efficacy, for example, heater probe or argon plasma coagulation. To try to achieve a similar benefit in everyday practice, one also has to consider logistical issues. The timing of colonoscopy within 6-12 hours of admission requires an out of hours endoscopy service, which in the UK is not universally available. It requires the presence of a trained endoscopist for accurate diagnosis and effective intervention, not forgetting the necessary assistant support and facilities. Out of hours colonoscopy would further increase the workload of already over stretched gastroenterologists.

The number of patients in the study was small and relied on historical controls. Further data are needed from larger randomised trials. The effectiveness and safety of urgent diagnostic and therapeutic endoscopy in lower 
gastrointestinal bleeding after a large volume purge remains to be established.

Gastroenterology Unit, Royal Cornwall Hospital, Truro, Cornwall TR1 3Lf, UK

Royal Cornwall Hospital, Truro, Cornwall TR1 3LF, UK

Correspondence to: Dr J L H Wong. johnlhw@netscape.net

J L H WONG

H R DALTON
1 Jensen DM, Machicado GA, Jutabha R, et al. Urgent colonoscopy for the diagnosis and treatment of severe diverticular hemorrhage. $N$ Engl f Med diagnosis and treat

2 McGuire HH Jr. Bleeding colonic diverticula. A reappraisal of natural history and management. Ann Surg 1994;220:653-6.

3 Longstreth GF. Epidemiology and outcome of patients hospitalised with acute lower gastrointestinal haemorrhage: a population-based study. $A m \mathcal{F}$ Gastroenterol 1997;92:419-24.

4 Farivar M, Perrotto JL. Urgent colonoscopy for the diagnosis and treatment of severe diverticular haemorrhage. N Engl f Med 2000;342:1609.

5 Jensen DM, Machicado GA. Diagnosis and treatment of severe haematochezia. The role of urgent colonoscopy after purge. Gastroenterology 1988; 95:1569-74. 